\title{
Consent, coercion and autonomy: Underage sex work in Aotearoa New Zealand
}

Natalie Thorburn University of Auckland, New Zealand

\begin{abstract}
INTRODUCTION: Adolescents' involvement in sex work in New Zealand has been associated with a range of adverse effects on physical, psychological, and social well-being, and is framed by domestic legislation and international obligations.
\end{abstract}

AIM: The study aimed to ascertain the nature of adolescents' experiences of sex work, and how their current and past environments impacted on their understanding of their involvement in sex work.

METHODS: Semi-structured interviews were used with a sample of eight adolescents aged 16-20 who became involved with sex work between ages 12 and 16 .

FINDINGS: The study found that participants' exposure to physical and sexual abuse in childhood had informed their internal working models of normative gendered behaviour. These models were then reinforced by exposure to the gendered power differential inherent in the sex work sub-culture. Participants experienced sexual experiences as being solely dictated by men, and frequently normalised transgressions against physical and/or sexual integrity.

CONCLUSION: The prevalence and acceptance of gendered harm among this population group indicates the need to appropriately address vulnerable adolescents' abuse histories, challenge restrictive conceptions of gender, and actively combat the pervasive presence of gendered violence and inequality among this population group.

KEY WORDS: underage sex work; abuse; violence; consent; adolescents; prostitution

AOTEAROA

NEW ZEALAND SOCIAL WORK 28(1), 34-42.

CORRESPONDENCE TO: Natalie Thorburn n.thorburn@auckland.ac.nz

\section{Introduction}

The phenomenon of underage sex work (USW) is often a polarising topic, as people's varied and often conflicting constructions of morality, gender, and social justice produce widely differing and often oppositional attitudes amongst researchers and practitioners. For the purposes of this research, young people/adolescents are considered to be people aged over 12 and under 18 years of age. Young people involved in sex work usually begin between the ages of 12 and 15 (Jordan, Patel, \& Kapp, 2013; Holger-Ambrose, Langmade, \&
Edinburgh et al, 2013; Lukman, Fauziah, \& Rusya et al, 2011; Chase \& Statham, 2005). The use of young people in sex work is often regarded as principally a child protection issue, despite significant divergence in the literature of accounts of the extent of choice and agency exercised by adolescents in sex work (Pearce, 2009).

According to a study conducted by ECPAT NZ, 194 children and young people aged under 18 years of age were known by social service professionals to be involved in USW in New Zealand in 2002 (Saphira, 2002). Such early involvement has been 
associated with exposure to physical and sexual violence, long-lasting psychological, physical, emotional, and cognitive impacts, including post-traumatic stress disorder, and a range of adverse physical health outcomes (Herman, 1987; Ahrens, Katon, \& McCarty et al, 2012). While the purchasing of sexual services from young people under the age of 18 is illegal in New Zealand, it is known to occur in a range of settings, and is viewed as an undeniable violation of children's rights within New Zealand's legislative framework despite the transactional nature of the offending (Ministry of Justice, 2002). However, attempts to investigate and target this phenomenon are hindered by the traumatic histories of adolescents using USW; the symptoms of which may perpetuate adolescents' involvement; adolescents' fear of stigma; and the largely hidden nature of the young person-client interactions (Saphira \& Oliver, 2002; Halter, 2010; Cusick, 2002).

A desperate need for income and a need for housing make young people extremely vulnerable to being inducted into underage sex work (Cobbina \& Oselin, 2011).

Approximately one in three young people in the United States will become involved with sex work within 48 hours of becoming homeless (Jordan, Patel, \& Rapp, 2013). Other studies discovered that between 20 and 50 percent of young people who become homeless will engage in underage sex work (Holger-Ambrose et al, 2013; Weber, Boivin, \& Blaise et al, 2002). Studies into economic deprivation and early entry into sex work have found that between 18 and 61.4 percent of participants identified their status of financial deprivation as being the chief motivator to entering sex work (Marcus, Horning, \& Curtis et al, 2014; Silbert, 1981).

Societal beliefs contribute to the continuation of young women's involvement in sex work. Oppressive patriarchal systems marginalise and sexualise women and children, increasing their experiences of maltreatment and resulting in unequal transactional power (Ashenden, 2004). Myths around men's uncontrollable sexual desires and the social ideal of women and girls as moral gatekeepers of sexuality, lead to a culture of silence and inaction about exploitative sexual situations (Saphira \& Oliver, 2002; Burnes, Schept, \& Long, 2012). Gendered messages to children groom them to be either victims or perpetrators, for example, by parents' encouraging types of gendered play that position girls as carers and boys as aggressive (Ennew, 1986). The grooming of female children to be submissive, together with the view that dominance and aggression are synonymous to masculinity, conditions children to exaggerate and continue these gendered behaviours through to adulthood (Dorais \& Corriveau, 2009). This forms the basis for male perpetration and female acceptance of the denigration of women (Dorais \& Corriveau, 2009). Adolescent girls are also sexualised very early in their dress style and behavioural expectations, in line with the increasingly popular ideal of young, newly developed adolescent girls as the ultimate sex objects (Dorais \& Corriveau 2009; Odone, 2010), which young women conform to (albeit at varying degrees) in order to gain social capital.

Within peer groups, continuous exposure to sexualised images in the media combined with the perceived connection between 'coolness' and sexual involvement has further entrenched unequal power dynamics in sexual relationships (Ministry of Women's Affairs, 2012). This has led to girls' levels of social inclusion being dependent on their having the 'right' level of sexual engagement, further contributing to girls' inferior positioning and consequent lack of choice when negotiating sexual relationships (Ministry of Women's Affairs, 2012).

Male violence against women and girls is normalised and often intergenerational, resulting in a deeply entrenched gendered power differential. (Pearce, 2009; Shannon, Kerr, Allinott, Chettiarr, Shoveller, \& Tindall, 2008). Further, the implicit blaming by the media of sex workers for their experiences of violence, reflect the widespread societal judgements that are made based on the supposed purity or impurity of female sex workers (Ministry of Women's Affairs, 2012). 
Child sexual abuse often precipitates early involvement in sex work. The relationship between sexual victimisation in childhood and later entry into USW is thought to occur through the changes to cognitive processes, relationships, perceptions of normative sexual behaviour, and psychological well-being resulting from abuse (Holger-Ambrose et al, 2013; Ahrens et al, 2012; Saphira \& Herbert, 2004a; Saphira \& Oliver, 2002). Survivors often experience substantial loss of self-worth following abuse (Shaw \& Butler, 1998), and traumatic responses may be reactivated if the re-enactment of sexual acts without free consent replicates the dynamics of the initial sexual abuse (Stebbins, 2010). Feelings of stigmatisation and shame beginning at the initial victimisation may also be reinforced by the act of selling sex (Ahrens, Fredlund, \& Goransvedin et al, 2012). In addition, one study into the feelings of young people involved with USW after their childhood abuse experiences showed that 55 percent felt disgusted by sexual activity, indicating a potential connection between child sexual abuse and latent distortions of victims' perceptions of sexuality, leading to the commodification of sex (Silbert, 1981).

Previous studies have shown that the prevalence rates of child sexual abuse among adolescent sex workers ranges from 40 to 60 percent, and this is likely to be a conservative statistic, given the inherent barriers to disclosure (Svensson et al, 2012; Cobbina \& Oselin, 2011; Saphira and Herbert, 2004a; Silbert, 1981). Finally, there is some evidence that sexual abuse may effectively condition young people for sex work, as through the abuse and reward patterns played out between the perpetrator and victim, children may begin to form associations between sex and the subsequent gaining of tangible rewards (Stebbins, 2010). This study was aimed at exploring and developing knowledge on adolescents' perceptions of their involvement in sex work, and on how their experiences in childhood and adolescence shaped their understanding of this involvement. Finally, it sought to identify ways in which harm relating to this involvement could be mitigated by appropriate intervention.

\section{Method}

A qualitative approach was used to collect and analyse data. Unlike quantitative deductive approaches, where theory contributes to the design of qualitative research, qualitative research is inductive and does not aim to predict the findings (Flick, 2011). Qualitative approaches also provide for the analysis of minute detail that is fundamentally valuable when examining or evaluating social variables (Alasuutari, 2010), and can therefore be considered to have a type of rigor absent in quantitative studies (Davis, 2007). The hidden and secretive nature of USW make the findings of research into their experience unpredictable. Conducting research with this population group qualitatively, provided the opportunity for participants to describe their experiences according to what they view as important.

Convenience sampling was used to recruit research participants, for reasons of both appropriateness and necessity. Convenience sampling is the process by which subjects are included based on both their appropriateness for the study and their accessibility (Morse, 2004). This entailed youth-focussed agencies displaying recruitment flyers at their premises. Prospective participants were excluded if they were deemed to be at imminent risk, resulting in a total of eight participants. The eight participants were all aged 16-20, and had been involved with USW prior to turning 18. Seven of the participants were female, and one was male. No transgender young people expressed interest in participating. Six of the participants identified as being Pākehā, and the remaining two identified as being New Zealand Māori.

The selection of participants aged 20 and under only, despite the limitation this 
imposed on sample size, was enforced so that the experiences analysed would be relevant to current policy frameworks. In addition, due to the re-formulation and re-contextualisation that retrospective remembering of experiences invariably involves, the perspectives of adolescents were considered more likely to provide insight into the meanings, they attributed to their experiences within a shorter time-frame of these occurring.

Semi-structured interviews were conducted with participants. Interview questions were designed with the assistance of specialists in related fields, and interviews ranged from 40 minutes to one hour in length, depending on the extent to which new data continued to emerge. Participants determined the pace of the interviews, and were asked questions about their childhood memories, families, current situations, and sex work involvement.

In the data reduction phase, the transcripts and memos were read several times to facilitate familiarity with the data. Next, the relevant words, phrases, and sections of the transcript were coded, which was aided by the use of colour coding, notes of observations, and diagrams, a useful strategy to bring order to the analytic process as suggested by Elo and Kyngas (2008). Coding was followed by the thematic organisation of words, phrases and sections denoting similar codes, which were then synthesised into broader categories as explained by Webb (1999) and visually mapped. Themes and patterns were observed from the combined results, and this was done in two stages; firstly, with only explicit content, and secondly, including interpretive findings (Paton, 2002). Finally, data was interpreted by the identification of patterns and key points arising from the coding (Elo \& Kyngas, 2008).

Approval was gained from the University of Auckland Human Participants Ethics Approval Committee on May 21, 2014 for a period of three years.

\section{Findings}

Power structures existed in some participants' lives that meant that sexual decision-making rights lay with others, rather than themselves. Known males were viewed as 'nice' despite coercing participants into sex, and sexual relationships between adults and minors (young people under the age of 18) were not typically viewed as abnormal or indicative of harmful behaviours. In the quotations that follow all participants have been given fictional names.

When I met Max, he was, like, real nice... he was the first guy I fucked. Uhh he told me I was pretty and stuff and made me feel good about my body, even though he was like 20, and I was like 11. (Participant \#2)

Female participants universally regarded the initiation of sex to be a male's prerogative.

I don't even remember the first time, it was just a bunch of times that it happened, by guys who just made me, you know. I never wanted it, it was just something that happened. (Participant \#4)

I saw sex as something that I didn't get to choose, someone else chose. (Participant \#6)

In addition, participants considered less 'serious' violence to be a natural part of sex work, despite working largely autonomously without 'pimps' or managers. Clients' preferences for strangling and choking workers were tolerated without complaint, despite physical injuries and fears for physical integrity. Both male and female participants disclosed this type of violence, often from their repeat clients who paid extra after causing pain or injury.

There was this one time where this guy said he'd give me extra if he could tie like a knot thing round my neck, so I agreed cos I knew Max would be pleased with the extra money. But, but he like tied it too tight and I was choking, that was 
pretty shit, I was so freaked out. Jesus Christ. And he got off of it, like came after a second while I wasn't breathing. (Participant \#2)

Physical violence within intimate relationships was rarely questioned or seen as unacceptable. A lack of caring others and the consequent emotional vulnerability of participants appeared to contribute to their tolerance of mistreatment, as violence seemed to be the preferred option compared to being alone or in state care. Three had regular partners who were physically violent, and the reasons given for the violence were jealousy, the participants' involvement in sex work, and control. Two blamed themselves for the violence, believing that it was unintentional and that if they had been better partners there would be no cause for violence. Jealousy in particular was often constructed as love.

He loved me too though, like he got real worked up if he thought someone was gonna take me away from him. He punched a hole in the wall once when he thought someone was hitting on me. -Participant \#4

When I told him, I thought he'd be happy, you know? Cos we'd talked about kids and stuff. It was about a year ago, I was 15. But he wasn't happy, he was really angry, and he smashed me in the tummy, like over and over, until I started bleeding, I lost the baby. And the guys, they were trying to get him to stop, and saying bro, bro, stop hitting your missus. And I thought they were like trying to protect me, but they were actually just, like, worried that the cops might come if I didn't stop screaming. (Participant \#2)

Although only half of the participants actually disclosed sexual abuse (and two adamantly explained that there had never been sexual abuse) all of the females interviewed described sexually abusive experiences, which had been normalised within their social environments. For example, one participant spoke about a sexual relationship that began when she was 11 with an adult male who was a friend of her father, and referred to this as a 'love' relationship. Participants who explicitly disclosed child sexual abuse made clear links between the abuse, their attitudes and beliefs around sexuality, and their involvement in sex work.

Sex has always been something that I've given in return for something else. When I was 16 or 17 then it was just for alcohol, or drugs. Even when I was a kid, like when I was nine, I was sexually abused by my uncle and he used to buy me things, take me shopping, for doing what he wanted and keeping quiet. (Participant \#3)

They also believed there were connections between the operative defence mechanisms they employed to deal with sexual abuse, and the methods that enabled them to distance themselves from the sexual act while working, such as dissociation and mental distraction.

It wasn't like having sex for cash was any different than being raped by my cousin. It felt exactly the same as it did when my cousin had sex with me. Or when my boyfriend did, or the business suit guys, or the cops. It was all the same. I didn't feel any of it. (Participant \#4)

It prepares you for sex work, it makes it not such a big impact as it if was someone who hadn't been abused going into that environment, you know. (Participant \#3)

Three also associated rape and sexual abuse with later lack of regard for self, and involvement in risky behaviour.

I probably wouldn't have been so... uncaring, so reckless... about myself, or my life, if I hadn't been [raped]. So I don't know if I would have been depressed, or anxious, or, like, smoked drugs or taken the kind of risks that I did. (Participant \#6) 
Participants also had varied conceptualisations of consent. Unwanted sexual acts were only deemed to be abusive if they involved penetration. The one incident that featured both violence and penetration was the only sexual incident that was clearly defined as rape, and the participant then rescinded this label as she spoke about her inaction at the time. Sexual encounters between participants and people in positions of power were also normalised and not framed as abusive (such as sexual encounters with police officers in exchange for money).

The high prevalence of early sexual victimisation among participants was consistent with the high rates documented in previous literature (Ahrens et al, 2013; Holger-Ambrose et al, 2013; Saphira \& Herbert, 2004a; Stebbins, 2010; Jordan, Patel, \& Rapp, 2013). Sexual experiences entailing coercive or violent elements were not framed as abusive, suggesting a distortion to participants' beliefs about acceptable sexual behaviour that has been identified in previous studies (Ahrens et al, 2012; Saphira \& Oliver, 2002). The pathway from early abuse to later involvement in USW appeared to derive from both direct and indirect channels, supporting the theory that USW may serve as a continuation of initial victimisation (Jordan, Patel, \& Rapp, 2013). In addition, participants associated the transactional or bargained nature of early sexual abuse with their willingness to agree to later transactions involving sex for money or goods, particularly when the childhood abuse featured tangible rewards from the perpetrator. This echoes Stebbins' (2010) assertion that sexual abuse in childhood effectively conditions young people for sex work through the initiation of a submission/ reward pattern.

Participants rarely considered the notion of free consent, instead equating their acceptance of payment from men or the existence of an intimate partner relationship with unconditional and automatic consent. The nature of sex work may serve as a continual reinforcement of this unequal dynamic, as male clients hold the balance of transactional power (Whibly, 2001). As a consequence, participants' autonomy was restricted by a gendered power differential, modelled to participants in childhood and reinforced within the sex work sub-culture to which they then belonged. This power differential precluded the development of bodily agency that would enable them to exercise unconstrained free choice in sexual decision-making.

Participants showed evidence of gendertyping and adherence to gendered beliefs, such as the assumption that men are inevitably violent and have sole decisionmaking power regarding the initiation of sex, and that women's needs are secondary to men's, which are similar to gendered beliefs identified in other studies into USW (Saphira \& Oliver, 2002; Burnes et al, 2012). Odone (2010) posits that such beliefs are derived from the dominant discourses within the immediate environment, primarily families and communities. The prevalence of these beliefs within the participant sample suggests widespread gender-typing and gender inequality within the sub-culture to which participants belong, and, quite possibly, the wider community. Gendered beliefs, and particularly those featuring rigid gender role adherence, have been linked to the perpetration of male violence against women (Burnes et al, 2012). The phenomenon of management by 'pimps' and accompanying violence or exploitation noted by multiple authors (Dorais \& Correvieau, 2009; Chase \& Statham, 2005; Pearce, 2009; Jordan, Patel, \& Rapp, 2013; Cobbina \& Oselin, 2011; Kennedy, 2011) was rare within the sample. However, male violence was clearly prevalent within participants' disclosed experiences. For example, female participants' descriptions of their interactions with males indicated a large degree of acceptance of violence and mistreatment by men, while abusive actions were clearly defined as abusive by the sole male participant. Violence perpetrated by men, regardless of whether it occurred in the context of sex work or intimate partner 
relationships, was not regarded as criminal or unusual, as illustrated by the stories of strangling, choking, and punching.

Tharp (2012) considers adolescent girls' acceptance of male violence to be influenced by both individual factors, such as negative self-concept and the endorsement of traditional gender roles; and relational factors, such as peer group influence, exposure to male aggression, and observation of violent conflict resolution processes of parents. These factors can all be identified in the lives of female participants, and appear to operate synergistically to ensure the continued tolerance of male violence. Female participants uniformly showed negative self-concept typically derived from childhood (often gendered) experiences of maltreatment. Correspondingly, messages about gender roles and identity that are received from early abusive experiences are believed to establish rigid internal working models about gender and consequent normative ideals about male and female behaviour (Scharf \& Maysleless, 2007; Dorais \& Corriveau, 2009). The combination of negative self-concept and personal adherence to stereotyped gender roles therefore creates vulnerability at the individual level, while the saturation of gendered beliefs and gendered violence within participants' environments reinforces this at the relational level. The witnessing of intimate partner violence between parents and subjection to sexual abuse from male family members further fortifies these gendered schemas, which then shape future expectations about malefemale interactions (Burnes et al, 2012; Shannon et al, 2008). The physical and sexual violence witnessed and experienced in childhood by participants is therefore likely to have independently contributed to their normalisation of victimisation by intimate partners, strangers, and clients in adolescence. Moreover, given the similarities in early experiences between participants, constructions of gender are likely to be essentially traditional, with this homogeneity of gender schemas being reinforced through peer contact.

\section{Conclusion}

From the results of this study, it is apparent that the gendered power differential modelled to participants in this sample was acutely manifest in multiple domains of their lives, including their experiences of sex work and their intimate relationships. To moderate the impact of this conditioning on future gendered expectations, abuse histories should be comprehensively addressed through trauma-focussed interventions, and sexuality and consent education programmes targeted to high-risk groups. The prevalence and normalisation of intimate partner violence should be actively combated at individual, community, and societal levels, and professions educated about the implicit messages that child sexual abuse and the witnessing of intimate partner violence transmits to children. Finally, the pervasive presence of gendered inequality needs to be addressed through universal public policy and health promotion awareness-raising approaches.

This study was limited by its comparatively small sample size and by the lack of diversity in participants' of gender and culture. In addition, it was intended to provide a broad overview of the experiences and perceived needs of underage sex workers; consequently, there was insufficient scope to fully explore the early caregiving experiences of the sample and how they contributed to the internalisation of gendered norms. Future studies in this area could seek to ascertain the impacts of specific early caregiver interactions and their effects on personal constructions of gender and conceptualisation of gendered violent behaviours. Finally, it would be useful to study the effects of targeted sexuality and consent education programmes on this population group to explore potential changes to their conceptualisations of gender, violence, and bodily sovereignty. 


\section{References}

Ahrens, K. R., Katon, W., McCarty, C., Richardson, L P., \& Courtney, M. E. (2012). Association between childhood sexual abuse and transactional sex in youth aging out of foster care. Child Abuse and Neglect, 36(1), 75-80.

Alasuutari, P. (2010). The rise and relevance of qualitative research. International Journal of Social Research Methodology, 13(2), 139-155.

Ashenden, S. (2004). Governing child sexual abuse: Negotiating the boundaries of public and private law and science. London, UK: Routledge.

Ayre, P., \& Barrett, D. (2000). Young people and prostitution: An end to the beginning? Children and Society, 14, 48-59.

Boeije, H. (2002). A purposeful approach to the constant comparative method in the analysis of qualitative interviews. Quality and Quantity, 36, 391-409.

Burnes, T R., Schept, R. A., \& Long, S. L. (2012). A resilience-based lens of sex work: Implications for professional psychologists. Professional Psychology: Research and Practice, 43(2), 137-144.

Chase, E., \& Statham, J. (2005). Commercial and sexua exploitation of children and young people in the UK: A review. Child Abuse Review, 14, 4-5.

Cobbina, J. E., \& Oselin, S. S. (2011). It's not only for the money: An analysis of adolescent versus adult entry into street prostitution. Sociological Inquiry, 81(3), 310-232.

Cusick, L. (2002). Youth prostitution: A literature review. Child Abuse Review, 11, 230-251.

Davis, N. (1981). Prostitutes. In G, Scrambler \& A. Scrambler, A. (Eds.), Deviance: the interactionist perspective. London, UK: Routledge.

Dorais, M., \& Corriveau, P. (2009). Gangs and girls: Understanding juvenile prostitution. Montreal, Canada: McGill Queen's University Press.

Elo, S., \& Kyngas, H. (2007). The qualitative content analysis process. Journal of Advanced Nursing, 62(1), 107-115.

Ennew, J. (1986). The sexual exploitation of children. Cambridge, UK: Polity Press.

Flick, U. (2014). An introduction to qualitative research (5th ed.). London, UK: Sage Publications Inc.

Fredlund, C., Svensson, F., Svedin, C. G., Priebe, G., \& Wadsby, M. (2013). Adolescents' lifetime experience of selling sex: Development over five years. Journal of Child Sexual Abuse, 22(3), 312-325.

Halter, S. (2010). Factors that influence police conceptualisations of girls involved in prostitution in six US cities: Child sexual exploitation victims or delinquents? Child Maltreatment, 15, 152-150.

Herman, K. J. (1987). Children sexually exploited for profit: A plea for a new social work priority. Social Work, 32(6), 523-525.

Holger-Ambrose, B., Langmade, C, Edinburgh, L., \& Saewyc, E. (2013). The illusions and juxtapositions of commercial sexual exploitation among youth: identifying effective street outreach strategies. Journal of child sexual abuse, 22(3), 326-340.

Hsieh, H. F., \& Shannon, S. E. (2005). Three approaches to qualitative content analysis. Qualitative Health Research, 15(9), 1277-1288.
Jordan, J., Patel, B., \& Rapp, L. (2013). Domestic minor sex trafficking: A social work perspective on misidentification, victims, buyers, traffickers, treatment, and reform of current practice. Journal of Health Behaviour in the Social Environment, 23(3), 356-367.

Kennedy A., Klein, C., Bristowe, J., Cooper, B., \& Yuille, J. (2007). Routes of recruitment: pimps' techniques and other circumstances that lead to street prostitution. Journal of Aggression, Maltreatment, and Trauma, 15(2), 1-19.

Lukman, Z. M., Fauziah, I., Rusyda, H. M., Sarnon, N., Mokhtar, M. K., Alavi, K.,...Fatimah, D. (2011). Betrayal of trust: The involvement of children in prostitution. Journal of Social Sciences and Humanities, 19(5), 49-56.

MacDonald, J-A., Gagnon, A. J., Mitchell, C., Megglio, G. D., Rennick, J. E., \& Cox, J. (2011). Asking to listen: towards a youth perspective on sexual health education and needs. Sex Education, 11(4), 443-457.

Macdonald, J., \& Morley, I. (2001). Shame and non-disclosure: A study of the emotional isolation of people referred for psychotherapy. British Journal of Medical Psychology, 74, $1-21$.

Marcus, A., Horning, A., Curtis, R., Sanson, J., \& Thompson, E. (2014). Conflict and agency among sex workers and pimps: A closer look at domestic minor sex trafficking. The ANNALS of the American Academy of Political and Social Science, 653(1), 225-246.

McNally, R. J., Bryant, R. A., \& Ehlers, A. (2003). Does early psychological intervention promote recovery from posttraumatic stress? Psychological Science in the Public Interest, 4(2), 45-80.

Ministry of Justice. (2002). Protecting our innocence: New Zealand's national plan of action against the commercial sexual exploitation of children. Wellington, New Zealand: Author. Retrieved from http://www.justice.govt.nz/publications/publicationsarchived/2002/protecting-our-innocence/documents/ final-plan.pdf

Ministry of Women's Affairs. (2012). Lightning does strike twice: Preventing sexual revictimisation. Wellington, New Zealand: Author

Morse, J. M. (2004). Constructing qualitatively derived theory. Journal of Qualitative Health Research, 14, 1387-1395.

Odone, C. (2010). Well done, Mumsnet, for campaigning against gross 'sexy' products for little girls. Retrieved from http://blogs.telegraph.co.uk/news/cristinaodone/ 100034558/well-done-mumsnet-for-campaigningagainst-gross-sexy-products-for-little-girls/

Patton, M. Q. (1999). Enhancing the quality and credibility of qualitative analysis. Health Services Research, 34(5), 1189-2000.

Patton, M. Q. (2002). Qualitative research and evaluation methods. Thousand Oaks, CA: Sage.

Pearce, J. (2009). Young people and sexual exploitation: 'It's not hidden, you just aren't looking'. New York: Routledge-Cavendish.

Saphira, M. (2002). Child prostitution in New Zealand Social Work Now, 22, 6-11

Saphira, M., \& Herbert, A. (2004). Exiting commercial sexual activity. Auckland, New Zealand: ECPAT NZ.

Saphira, M., \& Oliver, M. (2004). Alcohol and drug use among people who began sexual commercial activity before 18 years old. Auckland, New Zealand: ECPAT NZ. 
Saphira, M., \& Oliver, P. (2002). A review of literature on child prostitution. Social Policy Journal of New Zealand, 19, 141-163.

Scharf, M., \& Mayseless, O. (2007). Putting eggs in more than one basket: a new look at developmental processes of attachment in adolescence. New directions for child and adolescent development, 117, 3-22.

Shannon, K., Kerr, T., Allinott, S., Chettiar, J., Shoveller, J., \& Tyndall, M. W. (2008). Social and structural violence and power relations in mitigating HIV risk of drugusing women in survival sex work. Social Science and Medicine, 66, 911-921.

Silbert, M. H. (1981). Sexual child abuse as an antecedent to prostitution. Child Abuse and Neglect, 5, 407-411.

Skybo, T., \& Polivka, B. (2006). Health promotion model for childhood violence prevention and exposure. Journal of Clinical Nursing, 16, 38-45.

Speizer, I. S., Pettifor, A., Cummings, S., MacPhail, C., Kleinschmidt, I., \& Rees, H. V. (2009). Sexual violence and reproductive health outcomes among South African female youths: A contextual analysis. American Journal of Public Health, 99(2), 425-431.

Stebbins, J. P. (2010). Implications of sexuality counselling with women who have a history of prostitution. The Family Journal: Counselling and Therapy for Couples and Families, 18(1), 79-83.

Svensson, F., Fredlund, C., Goransvedin, C., Priebe, G., \& Wadsby, M. (2012). Adolescents selling sex: Exposures to abuse, mental health, self-harm behaviour, and the need for help and support - a study of a Swedish national sample. Informa Healthcare, 581-585.

Tharp, A. T. (2012). Dating matters: The next generation of teen dating violence prevention. Prevention Science, 13, 398-401

Vagi, K. J., Rothman, E. F., Latzman, N. E., Tharp, A. T., Hall, D. M., \& Breiding, M. J. (2013). Beyond correlates: A review of risk and protective factors for adolescent dating violence perpetration. Journal of Youth Adolescence, 42, 633-649.

Webb, C. (1999). Analysing Qualitative Data: computerised and other approaches. Journal of Advanced Nursing, 29(2), 323-330.

Weber, A. E., Boivin, J-F., Blais, L., Haley, N., \& Roy, E. (2002). HIV risk profile and prostitution among female street youths. Journal of Urban Health: Bulletin of the New York Academy of Medicine, 79(4), 525-535.

Whibly, J. (2001). This is work not your private life: A discourse analytic study of sex work in New Zealand. Massey University: New Zealand.

White, M. D., \& Marsh, E. E. (2006). Content analysis: A flexible methodology. Research Methods, 55(1), 22-45.

Wukzyn, F., Barth, R. P., Yuan, Y. Y. T., Harden, B. J., \& Landsverk, J. (2005). Beyond common sense: child welfare, child well-being, and the evidence for policy reform. New Brunswick, USA: Aldine Transaction. 